\title{
471338 - THE INCIDENCE OF PREOPERATIVE ANXIETY IN NEUROSURGICAL PATIENTS UNDERGOING A CRANIOTOMY
}

\author{
Anna Perks, MB ChB, FRCA, Sucharita Chakravarti, MD, Pirjo Manninen, MD \\ FRCPC, Lakshmikumar Venkat Raghavan, MD FRCA \\ Anesthesia, Toronto Western Hospital, University Health Network, University of \\ Toronto, Toronto, ON, Canada
}

Introduction: Preoperative anxiety is a common occurrence in many patients with different factors contributing to it. Patients with intracranial lesions may have additional preoperative fears due to the nature of their disease and procedure. This anxiety may impact on the anesthetic management of the patient. There is little information on incidence and severity of anxiety in patients scheduled for a craniotomy. The purpose of this study was to assess and compare the incidence and degree of preoperative anxiety between female $(\mathrm{F})$ and male $(\mathrm{M})$ patients undergoing a craniotomy.

Methods: Following IRB approval and written informed consent, 100 patients booked for a craniotomy were interviewed preoperatively in the preoperative anesthesia clinic or on the ward. Each patient was asked to grade their overall preoperative anxiety level on a verbal analog scale from 0 (no anxiety) to 10 (worse anxiety). A modified "Amsterdam Preoperative Anxiety and Information Scale” (APAIS) questionnaire and a set of specific anxiety related questions were conducted to assess the incidence of anxiety to specific factors(Table). (1) The anxiety scores and the responses to the questions were calculated and compared between the genders. Statistical analysis was with T-Test and Chi Square were appropriate and a $\mathrm{P}$ value $<0.05$ was considered significant.

Results: The mean age $( \pm S D)$ for F patients $(n=47)$ was $50 \pm 13$ and for $M(n=53) 50 \pm 14$ yr. The preoperative diagnosis was (F: M) tumor (28:36), aneurysm (6:8), and other (13:9). Anxiety scores were higher for F (5.8 \pm 2.8$)$ vs. M (4.6 \pm 2.5$)(p<0.05)$. Preoperative anxiety (worry) about anesthesia was present in $48 \%$ of all patients and about surgery in 87\%. Questionnaire results are in table.

Discussion: In our study preoperative anxiety regarding anesthesia and/or surgery was present in $89 \%$ of all patients. A previous study had shown that $60 \%$ of patients expressed anxiety prior to general surgical procedures (2). In this previous study (2) as well as in our study, female patients had greater anxiety compared to male. The areas of greatest concern included waiting for surgery and the surgical factors regarding the results of the operation and possible injury. Knowledge of the factors creating anxiety can be beneficial for improving perioperative patient care.

References: (1) Anesth Analg 1996;82:445-51 (2) Br J Anaesth 1967;39:503

Results: Anxiety scores and factors according to gender 


\begin{tabular}{|c|c|c|}
\hline APAIS scores ("0"-no anxiety-"6"-worse (mean \pm SD) & Female (n= 47) & Male (n=53) \\
\hline Worried about anesthesia & $1.9 \pm 2.0 *$ & $1.0 \pm 1.6$ \\
\hline Thinking about anesthesia & $1.4 \pm 2.0 *$ & $0.6 \pm 1.4$ \\
\hline Wants information about anesthesia & $2.7 \pm 2.3$ & $2.3 \pm 2.2$ \\
\hline Worried about surgery & $4.2 \pm 1.8 *$ & $3.2 \pm 2.0$ \\
\hline Thinking about surgery & $3.9 \pm 2.2$ & $3.2 \pm 1.9$ \\
\hline Wants information about surgery & $4.4 \pm 1.7$ & $3.7 \pm 2.2$ \\
\hline Percentage of patients who answered yes & & $62 \%$ \\
\hline Waiting for surgery & $77 \% *$ & $17 \%$ \\
\hline Being at mercy of staff & $36 \% *$ & $51 \%$ \\
\hline Not knowing what is happening & $74 \% *$ & $15 \%$ \\
\hline Discomfort from needles & $32 \% *$ & $25 \%$ \\
\hline Awareness during anesthesia & $57 \%$ & $28 \%$ \\
\hline Not awakening from anesthesia & $53 \% *$ & $77 \%$ \\
\hline Physical / mental harm & $80 \%$ & $23 \%$ \\
\hline Postoperative nausea/vomiting & $49 \%$ & $40 \%$ \\
\hline Postoperative pain & $85 \%$ & $81 \%$ \\
\hline
\end{tabular}

$*=\mathrm{P}<0.05$ from Male 